\title{
Iterative Receiver with Low Complexity for Downlink Multicarrier Communications over Rapidly Time-Varying Channels
}

\author{
Nihat Kabaoglu (iD, Eylem Erdogan (iD, and Erdogan Aydin (iD) \\ Department of Electrical and Electronics Engineering, Istanbul Medeniyet University, Istanbul, Turkey \\ Correspondence should be addressed to Nihat Kabaoglu; kabaoglunihat@gmail.com
}

Received 20 October 2017; Accepted 8 January 2018; Published 31 January 2018

Academic Editor: Simone Morosi

Copyright (C) 2018 Nihat Kabaoglu et al. This is an open access article distributed under the Creative Commons Attribution License, which permits unrestricted use, distribution, and reproduction in any medium, provided the original work is properly cited.

\begin{abstract}
This study proposes an iterative, joint channel estimation, equalization, and data detection method in the presence of high mobility for a multicarrier downlink system that communicates over rapidly time-varying channels. The proposed method uses a basis expansion method (BEM) which has low computational complexity and helps to reduce the number of coefficients needed to represent a time-varying channel and therefore is extremely easy to implement practically. Unlike the current literature, which is almost entirely focused on the uplink communication systems due to their computational costs, this method prioritizes the goal of being feasible in a downlink system with a reasonable performance. The proposed suboptimal algorithm is based on the space-alternating generalized expectation-maximization (SAGE) algorithm and the time-varying channel is represented by orthogonal basis functions obtained by means of discrete Walsh-Hadamard transform (DWHT). The resulting receiver iterates between maximum a posteriori (MAP) based channel estimation in the subspace spanned by the orthogonal basis functions and successive interference cancellation. Numerical examples show that the proposed algorithm has a satisfactory symbol error rate with low computational complexity and also has a reasonable peak-to-average power ratio (PAPR) reduction effect.
\end{abstract}

\section{Introduction}

1.1. Motivation and Previous Works. Multicarrier code division multiple access (MC-CDMA) combines the advantages of orthogonal frequency division multiplexing (OFDM) with code division multiple access (CDMA), especially partitioning the channel bandwidth into a series of parallel orthogonal subchannels. Thus, it turns frequency selective channel into a flat channel with one subcarrier. Each user's original data stream is spread across all subchannels by means of users' unique spreading codes in the frequency domain that separate users from each other. MC-CDMA thus provides frequency diversity and multiple access capability. This makes the MC-CDMA be a good candidate for these types of systems as well as OFDM. In the last two decades, the MCCDMA technique has been a powerful alternative to conventional modulation techniques for downlink systems since its spectral efficiency is high and its receiver complexity is low [1]. Today, it is still preferred as a popular multiple access technique for next generation wireless communication systems [2-5]. In multicarrier systems, if the symbol duration is smaller than the channel delay, multipath propagation causes serious multiple access interference (MAI) and intersymbol interference (ISI). The effects of MAI can be partially removed by using orthogonal codes, but it is not possible to completely remove it because of the deterioration on the orthogonality of the user codes and time delays. The adverse effects of MAI can be further reduced using estimates of all active users' data and channel coefficients. With this motivation, researchers studied iterative parallel interference canceller- (PIC-) based multiuser data detection techniques for CDMA-based systems to enhance their performance [611]. That is why maximum likelihood (ML) based algorithms such as expectation-maximization (EM) and spacealternating generalized expectation-maximization (SAGE) are preferred [11-14]. Although ML type algorithms are able to collectively estimate data and channel coefficients, they have computational complexity and slowness problem. To 
this end, these algorithms were mostly used in the uplink communication of multicarrier systems in [9-12] and rarely in downlink communication systems for data detection, channel estimation, beamforming, and direction of arrival estimation with a cost of high computational complexity [1419]. Despite high computational complexity, some of these studies show that jointly estimate of channel coefficients and user's data is a good alternative for the downlink communication of MC-CDMA system to improve the performance. Consequently, the joint data and channel estimation can be considered in downlink MC-CDMA systems to decrease computational complexity.

In order to meet the high data rate requirement of next generation communication systems, multicarrier modulation methods have become more attractive lately. Because the IEEE 802.11 standard series cannot perform satisfactorily for outdoor broadband wireless communications, the IEEE $802.16 \mathrm{~m}$ standard has been developed to address this need. As mobility is a very important issue in wireless environments, the studies on the channel estimation or/and the data detection for time-varying fading channels were considered in [13, 20-24]. Reference [13] presents a suboptimal receiver for OFDM systems where mobility is very high. It exploits discrete cosine transform (DCT) as basis expansion model instead of using whole channel and it has a remarkable performance. However, the computational complexity is still high. In [20], frequency shifted discrete prolate spheroidal (DPS) sequences are exploited to get an iterative time-variant channel estimator which does not need the detailed autocorrelation function of the channel. The proposed method in [21] is capable of controlling error propagation robustly and detecting and tracking abrupt channel changes effectively. Reference [22] proposes an iterative SAGE algorithm to jointly estimate the channel and data by using DPS sequences in timevarying flat-fading channels and [23] deals with estimation of rapidly time-varying Rayleigh fading channels. Specifically, it proposes a high order autoregressive (AR) model to track the channel, but it requires high computational cost when AR model order increases. The method in [24] proposes a time-frequency based channel estimator for MIMO-OFDM systems. In that paper, discrete evolutionary transformation is exploited to estimate doubly selective channel, but it has very high computational cost.

Since mobility is still important for the next generation systems as well as the current generation systems, BEM-based studies are still being carried out by many researchers. Some of the most remarkable of these studies can be found in [25-28]. Reference [25] presents an iterative receiver which uses two tolls: block-sparse-Bayesian learning and meanfield belief-propagation. The method has a satisfactory performance to estimate the delays, and their receiver performs almost excellently, but computational complexity of it is still an important problem since it uses a Bayesian based method. In [26], authors propose a channel estimation method using polynomial basis expansion method. It adaptively chooses the delay grid and iteratively estimates each path delay and then estimates path gains. Compared with the previous methods, it has much lower computational complexity as well as a comparable performance. In [27], a massive multiple-input multiple-output (MIMO) system with equipped antenna array is considered under the assumption of time-selective flat-fading channel. It presents a discrete Fourier transform based spatial-temporal basis expansion model to reduce number of channel coefficients. The remarkable aspect of this work is that the method can be applied to both FDD and TDD based massive MIMO systems, but its performance is severely affected since this method induces a large edge error and brings about the Gibbs phenomenon. In [28], authors investigate the performance of complex exponential BEM, generalized complex exponential BEM, and polynomial BEM while estimating the fast time-varying channel for a long term evolution for the railway system.

1.2. General Framework of Contributions. As can be seen above, all the aforementioned studies have considered detection and estimation methods for uplink communication systems due to high computational complexity. To the best of authors' knowledge, there is not any BEM-based study addressing the joint channel estimation, data detection, and equalization for downlink communication. Motivated above, in this paper, a low computational complexity SAGE-based iterative algorithm is proposed for downlink MC-CDMA system. The proposed method is capable of jointly estimating users' data and channel coefficients of rapidly time-varying and frequency selective channel. In order to represent timevarying fading channel low-dimensionally, basis function is obtained via DWHT. Instead of the channel coefficients, resulting low dimensional representations of them are estimated and then data symbols are detected iteratively with a reasonable computational complexity for the mobile user equipment. This is because DWHT needs simple addition and subtraction without the scaling factor. Hence, it is exploited in situations where the low computational burden is needed. Moreover, energy compaction rate of DWHT is at a reasonable level. Computer simulations show that DWHT shows a satisfactory performance in terms of symbol error rate.

Peak-to-average power ratio (PAPR) is one of the challenging issues in OFDM based systems. High PAPR force the high power amplifier to operate in its linear region with wide dynamic range, where the power efficiency is very poor. The poor power efficiency makes the reduction of PAPR more important in OFDM systems. In order to reduce this effect, various methods such as selected mapping, hierarchical QAM, wavelet transform, Karhunen-Loeve Transform, WHT, and double WHT have been proposed in [29-34]. Particularly, the studies using WHT in $[33,34]$ have attracted attention because of their superior performance. With this motivation, we investigate whether our proposed DWHT based method has any effect on reducing the PAPR.

1.3. Organization of the Paper and Notations. This paper is organized as follows. In Section 2, signal model of downlink MC-CDMA system for rapidly time-varying and frequency selective fading channels is described. Section 3 presents a suboptimal receiver consisting of a channel estimator and data detector with interference cancellation. Computer simulations are presented in Section 4. Finally, Section 5 concludes the paper. 
Notation 1. $(\cdot)^{-1},(\cdot)^{T},(\cdot)^{H}, \operatorname{trc}[\cdot], \otimes$, and $\mathfrak{R}(\cdot)$ denote matrix inversion, transpose, conjugate transpose, trace, Kronecker product, and real part of its argument, respectively. Vectors (matrices) are denoted by boldface lower (upper) case letters; all vectors are column vectors. $\mathbf{I}_{N}$ represents the $N \times N$ identity matrix and $\mathbf{1}_{N}$ is $N \times 1$ vector each element of which is 1 .

\section{Signal Model}

This study considers a synchronous downlink MC-CDMA system in which there are a simultaneously active $K$ number of users. Each user has a preassigned spreading code with length $P$. It is considered that the number of subcarriers $N$ is equal to $P$. In this system, each active user's data is first spread with its own unique codes, and then each chip of spread data symbol is mapped onto a different subcarrier. After the processes of mapping, $\mathrm{P} / \mathrm{S}$ conversion, and cyclic prefix (CP) insertion, the obtained signal is transmitted through a timevarying and frequency selective fading channel. Discretetime impulse response of the channel can be defined as $c(m, s)=\sum_{s=1}^{S} g_{s}(m) \delta\left(t-\tau_{s}(m)\right), s=0,1, \ldots, S-1, m=$ $0,1, \ldots, M-1$, where $S$ is the maximum channel length and $M$ is the length of one MC-CDMA frame, respectively; $g_{s}(m)$ and $\tau_{s}(m)$ are time-varying complex fading coefficient and delay of the $s$-th path, respectively. The channel vector is defined as $\mathbf{g}(m)=\left[\mathbf{g}_{0}^{T}(m), \mathbf{g}_{1}^{T}(m), \ldots, \mathbf{g}_{S-1}^{T}(m)\right]^{T} \in \mathbb{C}^{N S \times 1}$, where $\mathbf{g}_{s}(m)=\left[g_{s}(0), g_{s}(1), \ldots, g_{s}(N-1)\right]^{T}$ represents WSSUS Rayleigh fading coefficients of $s$-th path at $m$-th discrete-time. According to Jake's model, the autocorrelation function of the channel is

$$
\begin{aligned}
R_{g_{s}} & \left(m-m^{\prime}, s-s^{\prime}\right) \\
\quad & \sigma_{g_{s}}^{2} J_{0}\left(2 \pi f_{D} T_{s}\left(m-m^{\prime}\right)\right) \delta\left(s-s^{\prime}\right),
\end{aligned}
$$

where $\sigma_{g_{s}}^{2}$ denotes the normalized power of the channel coefficients of the $s$-th path. $f_{D}$ is the Doppler frequency in Hertz so that the term $f_{D} T_{s}$ represents normalized Doppler frequency where $T_{s}$ is sampling duration. As long as $f_{D} T_{s}$ is sufficiently small, impulse response of the time-varying channel can be regarded as constant throughout an MC-CDMA frame. $J_{0}(\cdot)$ is the zeroth-order Bessel function of the first kind.

After the sampling of the signal received by the $k$-th mobile unit at an adequate rate and S/P conversion, CP is removed. Later, the obtained discrete-time signal is demodulated by using DFT. Finally, the resulting signal for $m$-th symbol at the output of $k$-th mobile user's matched filter can be written as [14]

$$
y(m)=\sum_{n=1}^{K} b_{n}(m) \boldsymbol{\rho}_{n k} \mathbf{F}(m) \mathbf{g}(m)+w(m),
$$

where $b_{n}(m)$ is $m$-th symbol of $n$-th user; $\boldsymbol{\rho}_{n k}$ is the cross correlation among user's spreading codes with length $N$, which is $\boldsymbol{\rho}_{n k}=[1,1, \ldots, 1] \in \mathbb{R}^{1 \times N}$ when $n=k ; \mathbf{F}(m)=$ $\mathbf{1}_{N}^{T} \otimes \widetilde{\mathbf{F}} \in \mathbb{C}^{N \times N S}$ is the Fourier transform matrix for $m$-th symbol where $\widetilde{\mathbf{F}} \in \mathbb{C}^{N \times S}$ is with the $(n, s)$-th element given by $e^{-2 \pi p \tau_{s}(m) / N}$; and $w(m)$ is distributed as $\mathcal{N}\left(0, \sigma^{2} / N\right)$. After few manipulations, (2) can be written in a more compact form including all $M$ number of symbols in a frame as follows:

$$
\mathbf{y}=\mathbf{Q g}+\mathbf{w}
$$

Here, $\mathbf{y}=[y(0), y(1), \ldots, y(M-1)]^{T} \in \mathbb{C}^{M \times 1}$ is the signal vector obtained from output of $k$-th active user's matched filter, $\mathbf{Q}=\sum_{n=1}^{K} \mathbf{Q}_{n k}$, where $\mathbf{Q}_{n k}=\operatorname{diag}\left(\mathfrak{b}_{n}\right) \mathbf{A}_{n} \in \mathbb{C}^{M \times M N S}$ for $\mathbf{A}_{n}=\mathbf{I}_{M} \otimes \boldsymbol{\rho}_{n k} \mathbf{F}(m) \in \mathbb{C}^{M \times M N S}$ and $\mathfrak{b}_{n}=\left[b_{n}(1), \ldots, b_{n}(M)\right]^{T}$, and $\mathbf{w}=[w(0), w(1), \ldots, w(M-1)]^{T} \in \mathbb{C}^{M \times 1}$ is Gaussian noise vector distributed according to $\mathscr{N}\left(0,\left(\sigma^{2} / N\right) \mathbf{I}_{M}\right) . \mathfrak{b}_{n}$ is symbol vector sent by the $n$-th user within a frame period for $n=1,2, \ldots, k, \ldots, K$ and each symbol vector consists of $L$ number of pilot symbols and $M-L$ number of data symbols. The vector $\mathbf{g}$ denotes time-varying channel impulse response

$$
\mathbf{g}=\left[\mathbf{g}^{T}(0), \mathbf{g}^{T}(1), \ldots, \mathbf{g}^{T}(M-1)\right]^{T} .
$$

The mobile receiver's symbol detection performance is significantly dependent on the quality of the estimate of channel $\mathbf{g} \in \mathbb{C}^{M N S \times 1}$. However, it is not possible to directly obtain an estimate of the channel vector $\mathbf{g}$ by using received vector $\mathbf{y}$ since it has less number of equations than number of unknowns. In other words, this system is overdetermined. From $[7,8]$, it can be seen that most of the computational complexity in the symbol detection results from the channel estimation process. Since the rapidly time-varying and frequency selective channel is longer than that of considered in those works, channel estimation process has higher computational cost. In order to reduce complexity and to directly estimate the channel vector, a low dimensional subspace based approximation for the time-varying channel $\mathbf{g}$ should be regarded. Therefore, the channel coefficients $g_{s}(m)$ can be represented as a weighted sum of $M$ orthonormal basis functions $\left\{\mathbf{u}_{d}(m)\right\}$ in the interval $\left[0, M T_{s}\right]$ as follows:

$$
g_{s}(m)=\sum_{d=0}^{M N-1} u_{d}(m) h(d, s), \quad m=0,1, \ldots, M N-1,
$$

where $h(d, s)$ are the basis expansion coefficients. The bandwidth of $g_{s}(m)$ is $D \ll M N$ where it indicates the dominant number of eigenvalues determining the Doppler spectra. The dimension $D$ of subspace can be lower bounded by $D^{\prime}=\left\lceil 2 f_{D_{\max }} M+1\right\rceil$, where $f_{D_{\max }}$ the maximum normalized Doppler bandwidth is

$$
f_{D_{\max }}=\frac{v_{\max }}{c_{0}} f_{c} T_{s}
$$

$f_{c}, v_{\max }$, and $c_{0}$ are the carrier frequency, the maximum relative speed between the mobile and the base-station, and the speed of light, respectively. Then, $g_{s}(m)$ can be written as its own approximation:

$$
\bar{g}_{s}(m)=\sum_{d=0}^{D-1} u_{d}(m) h(d, s), \quad m=0,1, \ldots, M N-1 .
$$


Basis expansion coefficients can be obtained using the orthogonality property of the basis functions as follows:

$$
h(d, s)=\sum_{m=0}^{M N-1} u_{d}(m) g_{s}(m), \quad d=0,1, \ldots, D-1
$$

In this study, DWHT basis functions are exploited to represent the time-varying and frequency selective fading channel in a low dimensional subspace, which are given by

$$
u_{d}(m)=\operatorname{wal}(d, m)=\prod_{j=0}^{n} \operatorname{sgn}\left(\cos \left(d_{j} 2^{j} \pi m\right)\right),
$$

where the integer $d$ has the binary expansion

$$
d=d_{0} 2^{0}+d_{1} 2^{1}+d_{2} 2^{2}+\cdots+d_{n} 2^{n},
$$

and the binary coefficients $d_{j}$ are each 0 or 1 . The Walsh functions take only the values $\mp 1$ and they change sign only when $m$ is a multiple of a power of $1 / 2$. The index $d$ termed the sequence of a Walsh function, analogous to frequency in Fourier analysis; therefore DWHT basis functions are also capable of representing the low-pass equivalent of the channel. Number of the DWHT basis functions can be limited by choosing $D$ smaller than $M N$; thus mean square of the channel modeling error can be controlled. Channel and DWHT expansion coefficients for each channel path are connected to each other with the following equations:

$$
\begin{aligned}
& \mathbf{g}_{s}=\mathbf{U h}_{s}, \\
& \mathbf{h}_{s}=\mathbf{U}^{T} \mathbf{g}_{s},
\end{aligned}
$$

where

$$
\begin{aligned}
& \mathbf{g}_{s}=[g(0, s), g(1, s), \ldots, g(M N-1, s)]^{T} \in \mathbb{C}^{M N \times 1}, \\
& \mathbf{h}_{s}=[h(0, s), h(1, s), \ldots, h(D-1, s)]^{T} \in \mathbb{C}^{D \times 1}, \\
& \mathbf{U}=[\mathbf{u}(0), \mathbf{u}(1), \ldots, \mathbf{u}(M N-1)]^{T} \in \mathbb{R}^{M N \times D},
\end{aligned}
$$

with the basis functions at $m$-th sample time

$$
\mathbf{u}(m)=\left[u_{0}(m), u_{1}(m), \ldots, u_{D-1}(m)\right]^{T} \in \mathbb{R}^{D \times 1} .
$$

In light of the foregoing, for $m$-th sample of all channel paths, the channel and the expansion coefficients can be expressed in matrix form

$$
\mathbf{g}(m)=\mathfrak{U}(m) \mathbf{h}(m)
$$

where

$$
\mathcal{U}(m)=\operatorname{diag} \underbrace{(\boldsymbol{U}(m), \boldsymbol{U}(m), \ldots, \boldsymbol{U}(m))}_{S} \in \mathbb{R}^{N S \times D S},
$$

with $\boldsymbol{U}(m)=[\mathbf{u}(0), \mathbf{u}(1), \ldots, \mathbf{u}(N-1)]^{T} \in \mathbb{R}^{N \times D}$ and $\mathbf{h}(m)=\left[\mathbf{h}_{0}^{T}, \mathbf{h}_{1}^{T}, \ldots, \mathbf{h}_{S-1}^{T}\right]^{T} \in \mathbb{C}^{D S \times 1}$. Here, $\mathbf{h}_{s}$ denotes the channel coefficients for $s$-th path, which can be expressed as $\mathbf{h}_{s}=[h(0, s), h(1, s), \ldots, h(D-1, s)]^{T} \in \mathbb{C}^{D \times 1}$. After few manipulations, (14) can be arranged as follows:

$$
\mathbf{g}=\mathfrak{U} \mathbf{h},
$$

where $\mathfrak{U}$ represents modified DWHT matrix expressed as

$$
\begin{aligned}
\mathfrak{U} & =\operatorname{diag}(\mathfrak{U}(0), \mathfrak{U}(1), \ldots, \mathfrak{U}(M-1)) \\
& \in \mathbb{R}^{M N S \times M D S}, \\
\mathbf{h} & =\left[\mathbf{h}^{T}(0), \mathbf{h}^{T}(1), \ldots, \mathbf{h}^{T}(M-1)\right]^{T} \in \mathbb{C}^{M D S \times 1} .
\end{aligned}
$$

Finally, inserting (14) into (2), the received signal can be written as

$$
y(m)=\mathbf{x}(m) \mathbf{h}(m)+w(m)
$$

where $\mathbf{x}(m)=\sum_{n=1}^{K} b_{n}(m) \boldsymbol{\rho}_{n k} \mathbf{F}(m) \mathfrak{U}(m)$. Substituting (16) into (3), a more compact form of (18) can be obtained as

$$
\mathbf{y}=\Theta \mathbf{h}+\mathbf{w}
$$

where $\Theta=\mathbf{Q} \mathcal{U} \in \mathbb{C}^{M \times M D S}$.

\section{The Proposed SAGE-Based Receiver}

The problem at hand is to detect the user's data using the signal model in (19), without having full channel state information. The aim of this work is to derive an EM-based iterative algorithm fulfilling that need. This algorithm will be capable of cancelling the MAI as well as jointly estimating channel and detecting the data by decomposing the received signal in (19) into two summands that are noisy versions of the desired signal and MAI.

$$
\mathbf{y}=\underbrace{\Theta_{k} \mathbf{h}+\mathbf{w}}_{\mathbf{y}_{k}}+\underbrace{\Theta_{\mathrm{MAI}} \mathbf{h}}_{\mathbf{y}_{\mathrm{MAI}}}=\mathbf{y}_{k}+\mathbf{y}_{\mathrm{MAI}},
$$

where $\boldsymbol{\Theta}_{k}=\mathbf{Q}_{k k} \mathcal{U}$ and $\boldsymbol{\Theta}_{\mathrm{MAI}}=\sum_{n=1, n \neq k}^{K} \mathbf{Q}_{n k} \mathcal{U}$. Each element of $\mathbf{y}_{k}$ and $\mathbf{y}_{\mathrm{MAI}}$ can be written, respectively, as follows:

$$
\begin{aligned}
y_{k}(m) & =b_{k}(m) \boldsymbol{\rho}_{k k} \mathbf{F}(m) \mathfrak{U}(m) \mathbf{h}+w(m), \\
y_{\mathrm{MAI}}(m) & =\sum_{n=1, n \neq k}^{K} b_{n}(m) \boldsymbol{\rho}_{n k} \mathbf{F}(m) \mathfrak{U}(m) \mathbf{h} .
\end{aligned}
$$

Now, the SAGE algorithm can be used to detect each users' data vectors based on the received vector y. Firstly, the complete and incomplete data can be described. The suitable choices for complete data and incomplete data are $\boldsymbol{\chi}=\left\{\mathbf{y}_{k}, \mathbf{h}\right\}$ and $\mathbf{y}$, respectively. $\boldsymbol{\theta}=\mathfrak{b}$ is the parameter vector to be estimated. The likelihood function of the complete data can be written as

$$
\mathfrak{p}(\chi \mid \boldsymbol{\theta})=\mathfrak{p}\left(\mathbf{y}_{k}, \mathbf{h} \mid \mathfrak{b}\right)=\mathfrak{p}\left(\mathbf{y}_{k} \mid \mathbf{h}, \mathfrak{b}\right) \mathfrak{p}(\mathbf{h} \mid \mathfrak{b})
$$


Since $\mathbf{h}$ and $\mathfrak{b}$ are independent from each other, the last term in (22) is a constant. After neglecting the unnecessary terms, log-likelihood function can be written as follows:

$$
\begin{aligned}
\ln \mathfrak{p} & (\boldsymbol{x} \mid \boldsymbol{\theta})=\ln \mathfrak{p}\left(\mathbf{y}_{k} \mid \mathbf{h}, \mathfrak{b}\right) \\
\cong & \sum_{m=0}^{M-1}\left[\mathfrak{R}\left\{b_{k}^{*}(m) \boldsymbol{\kappa}^{H}(m) y_{k}(m)\right\}\right. \\
& \left.-\frac{1}{2}\left|b_{k}(m)\right|^{2} \boldsymbol{\kappa}^{H}(m) \boldsymbol{\kappa}(m)\right],
\end{aligned}
$$

where $\boldsymbol{\kappa}_{k}(m)=\boldsymbol{\rho}_{k k} \mathbf{F}(m) \mathfrak{U}(m)$.

3.1. Expectation Step. The aim of this step is to take the conditional expectation of log-likelihood function over $\chi$ when we have observed data $\mathbf{y}$ and an estimation of $\mathfrak{b}^{(j)}$. Assuming we have them, this expectation is given as

$$
\mathfrak{\Omega}_{k}\left(\mathfrak{b}_{k} \mid \mathfrak{b}^{(j)}\right)=E\left\{\ln \mathfrak{p}(\boldsymbol{x} \mid \boldsymbol{\theta}) \mid \mathbf{y}, \mathfrak{b}^{(j)}\right\} .
$$

Inserting (23) into (24), we have

$$
\begin{aligned}
\mathfrak{\Omega}_{k} & \left(\mathfrak{b}_{k} \mid \mathfrak{b}^{(j)}\right) \\
= & \sum_{m=0}^{M-1} \mathfrak{R}\left\{b_{k}^{*}(m) E\left\{\mathbf{h}^{H} \boldsymbol{\kappa}_{k}^{H}(m) y_{k}(m) \mid \mathbf{y}, \mathfrak{b}^{(j)}\right\}\right\} \\
& \quad-\frac{1}{2}\left|b_{k}(m)\right|^{2} E\left\{\mathbf{h}^{H} \boldsymbol{\kappa}_{k}^{H}(m) \boldsymbol{\kappa}_{n}(m) \mathbf{h} \mid \mathbf{y}, \mathfrak{b}^{(q)}\right\} .
\end{aligned}
$$

The first expected value in (25) can be written by using conditional expectation rule $E\left\{\mathbf{h}^{H} \boldsymbol{\kappa}_{k}^{H}(m) y_{k}(m) \mid \mathbf{y}, \mathfrak{b}^{(j)}\right\}=$ $E\left\{\mathbf{h}^{H} \boldsymbol{\kappa}_{k}^{H}(m) E\left\{y_{k}(m) \mid \mathbf{h}, \mathbf{y}, \mathfrak{b}^{(j)}\right\} \mid \mathbf{y}, \mathfrak{b}^{(j)}\right\}$ and then we have

$$
\begin{aligned}
E\left\{y_{k}(m) \mid \mathbf{h}, \mathbf{y}, \mathfrak{b}^{(j)}\right\} \\
=b_{k}^{(j)}(m) \boldsymbol{\kappa}_{k}(m) \mathbf{h} \\
\quad+\left(y(m)-\sum_{n=1}^{K} b_{n}^{(j)}(m) \boldsymbol{\kappa}_{n}(m) \mathbf{h}\right),
\end{aligned}
$$

where $\mathfrak{b}^{(q)}$ stands for the estimated value of signal $\mathfrak{b}$ at $q$-th iteration step. The second expected value in (25) can be obtained as

$$
\begin{array}{r}
E\left\{\mathbf{h}^{H} \boldsymbol{\kappa}_{k}^{H}(m) \boldsymbol{\kappa}_{n}(m) \mathbf{h} \mid \mathbf{y}, \mathfrak{b}^{(j)}\right\} \\
=E\left\{\mathbf{h}^{H} \boldsymbol{\Gamma}_{k n}(m) \mathbf{h} \mid \mathbf{y}, \mathfrak{b}^{(j)}\right\},
\end{array}
$$

where $\boldsymbol{\Gamma}_{k n}(m)=\boldsymbol{\kappa}_{k}^{H}(m) \boldsymbol{\kappa}_{n}(m)$. Using the results obtained by (26) and (27), (25) can be rewritten as

$$
\begin{aligned}
\mathfrak{Q}_{k}\left(\mathfrak{b}_{k} \mid \mathfrak{b}^{(j)}\right) & \\
= & \sum_{m=0}^{M-1} \mathfrak{R}\left\{b_{k}^{*}(m) \beta_{k}^{(j)}(m)\right\} \\
& \quad-\frac{1}{2}\left|b_{k}(m)\right|^{2} E\left\{\mathbf{h}^{H} \boldsymbol{\Gamma}_{k n}(m) \mathbf{h} \mid \mathbf{y}, \mathfrak{b}^{(j)}\right\},
\end{aligned}
$$

where

$$
\begin{aligned}
\beta_{k}^{(j)}(m)= & E\left\{\mathbf{h}^{H} \mid \mathbf{y}, \mathfrak{b}^{(j)}\right\} \boldsymbol{\kappa}^{H}(m) y_{k}(m) \\
& -\sum_{n=0, n \neq k}^{K-1} b_{k}^{(j)}(m) E\left\{\mathbf{h}^{H} \boldsymbol{\Gamma}_{k n}(m) \mathbf{h} \mid \mathbf{y}, \mathfrak{b}^{(j)}\right\} .
\end{aligned}
$$

In order to evaluate $E\left[\mathbf{h}^{H} \mid \mathbf{y}, \mathfrak{b}^{(j)}\right]$ and $E\left[\mathbf{h}^{H} \boldsymbol{\Gamma}_{k n}(m) \mathbf{h} \mid \mathbf{y}, \mathfrak{b}^{(j)}\right]$ in (29), $\mathfrak{p}\left(\mathbf{h} \mid \mathbf{y}, \mathfrak{b}^{(j)}\right)$ must be known. It can be obtained by using (3) as

$$
\mathfrak{p}\left(\mathbf{h} \mid \mathbf{y}, \mathfrak{b}^{(j)}\right) \cong \mathfrak{p}\left(\mathbf{y} \mid \mathbf{h}, \mathfrak{b}^{(j)}\right) \mathfrak{p}(\mathbf{h})
$$

After few manipulations, (30) can be written as

$$
\mathfrak{p}\left(\mathbf{h} \mid \mathbf{y}, \mathfrak{b}^{(j)}\right) \sim N\left(\boldsymbol{\mu}_{\mathbf{h}}^{(j)}, \boldsymbol{\Sigma}_{\mathbf{h}}^{(j)}\right),
$$

where

$$
\begin{aligned}
& \boldsymbol{\mu}_{\mathbf{h}}^{(j)}=\frac{N}{\sigma^{2}} \boldsymbol{\Sigma}_{\mathbf{h}}^{(j)} \Theta^{(j)}{ }^{H} \mathbf{y} \\
& \boldsymbol{\Sigma}_{\mathbf{h}}^{(j)}=\left[\mathbf{C}_{\mathbf{h}}^{-1}+\frac{N}{\sigma^{2}} \boldsymbol{\Theta}^{(j)^{H}} \boldsymbol{\Theta}^{(j)}\right]^{-1} .
\end{aligned}
$$

It is important to note that (32) is a MAP estimator of the channel. The matrix $\mathbf{C}_{\mathbf{h}}$ is the covariance matrix of $\mathbf{h}$. We assume that the vector $\mathbf{h}$ is distributed as $N\left(\mathbf{0}, \mathbf{C}_{\mathbf{h}}\right)$, where

$$
\mathbf{C}_{\mathbf{h}}=\operatorname{diag}\left(\mathbf{C}_{\mathbf{h}}(0), \mathbf{C}_{\mathbf{h}}(1), \ldots, \mathbf{C}_{\mathbf{h}}(S-1)\right) \in \mathbb{C}^{D S \times D S},
$$

with each diagonal matrix $\mathbf{C}_{\mathbf{h}}(s)=\mathbf{U}^{T} \mathbf{C}_{\mathbf{g}}(s) \mathbf{U}$. By using (1), the Toeplitz covariance matrix $\mathbf{C}_{\mathbf{g}}(s)$ can easily be expressed as

$$
\begin{aligned}
& \mathbf{C}_{\mathbf{g}}(s) \\
& =\sigma_{g_{s}}^{2}\left[\begin{array}{cccc}
c(0) & c(1) & \ldots & c(M N-1) \\
c(1) & c(0) & \ldots & c(M N-2) \\
\vdots & \vdots & \ddots & \vdots \\
c(M N-1) & c(M N-2) & \ldots & c(0)
\end{array}\right],
\end{aligned}
$$

with $c(k)=J_{0}\left(2 \pi f_{d} n T_{s}\right) . n$ represents the subcarrier index. If the length of the observation frame is large enough, then the covariance matrix $\mathbf{C}_{\mathbf{g}}(s)$ becomes diagonal for each channel path

$$
\mathbf{C}_{\mathbf{g}}(s)=\sigma_{g_{s}}^{2} \operatorname{diag}(\gamma(s, 0), \gamma(s, 1), \ldots, \gamma(s, D-1)) .
$$

Here, $\gamma(s, d)=S_{h}\left(s, d / 2 N M T_{s}\right)$ is the scattering function of the channel for $d=0,1, \ldots, D-1$, where each of them is the Fourier transform of $c(k)$. In [35], it is defined as $S_{h}(s, f)=$ $\sigma_{g_{s}}^{2} / \pi \sqrt{f_{D}^{2}-f^{2}}$ for $|f|<f_{D}$. The first expected value in (29) can be found as

$$
E\left\{\mathbf{h}^{H} \mid \mathbf{y}, \mathfrak{b}^{(j)}\right\}=\boldsymbol{\mu}_{\mathbf{h}}^{(j)} .
$$

The last expectation in (29) can be computed as

$$
E\left\{\mathbf{h}^{H} \boldsymbol{\Gamma}_{k n}(m) \mathbf{h} \mid \mathbf{y}, \mathfrak{b}^{(j)}\right\}=\operatorname{trc}\left[\mathscr{K}^{(j)}(m)\right],
$$


where

$$
\begin{aligned}
\mathscr{K}^{(j)}(m) & =E\left\{\boldsymbol{\kappa}_{n}(m) \mathbf{h h}^{H} \boldsymbol{\kappa}_{k}^{H} \mid \mathbf{y}, \mathfrak{b}^{(j)}\right\} \\
& =\boldsymbol{\kappa}_{n}(m)\left(\boldsymbol{\Sigma}_{\mathbf{h}}^{(j)}+\boldsymbol{\mu}_{\mathbf{h}}^{(j)} \boldsymbol{\mu}_{\mathbf{h}}^{(j)^{H}}\right) \boldsymbol{\kappa}_{k}^{H}(m) .
\end{aligned}
$$

Inserting (37) and (38) into (29), $\beta_{k}^{(j)}(m)$ can be obtained as

$$
\begin{gathered}
\beta_{k}^{(j)}(m)=\boldsymbol{\mu}_{\mathbf{h}}^{(j)^{H}} \boldsymbol{\kappa}_{k}^{H}(m) y_{k}(m)-\sum_{n=0, n \neq k}^{K-1} b_{k}^{(j)}(m) \\
\cdot \operatorname{trc}\left[\boldsymbol{\kappa}_{n}(m)\left(\boldsymbol{\Sigma}_{\mathbf{h}}^{(j)}+\boldsymbol{\mu}_{\mathbf{h}}^{(j)} \boldsymbol{\mu}_{\mathbf{h}}^{(j)^{H}}\right) \boldsymbol{\kappa}_{k}^{H}(m)\right] .
\end{gathered}
$$

3.2. Maximization Step. In this step, the proposed SAGE algorithm is executed to update the data vector $\mathfrak{b}$. It can be updated as follows:

$$
\mathfrak{b}_{k}^{(j+1)}=\arg \max _{\mathfrak{b}_{k}} \mathfrak{\mathfrak { L }}_{k}\left(\mathfrak{b}_{k} \mid \mathfrak{b}^{(j)}\right) .
$$

Inserting $\mathfrak{\mathfrak { L }}_{k}\left(\mathfrak{b}_{k} \mid \mathfrak{b}^{(j)}\right)$ in (24) into (41), we obtain the following:

$$
\begin{aligned}
\mathfrak{b}_{k}^{(j+1)}= & \arg \max _{\mathfrak{b}_{k}} \sum_{m=0}^{M-1} \mathfrak{R}\left\{b_{k}^{*}(m) \beta_{k}^{(j)}(m)\right\} \\
& -\frac{1}{2}\left|b_{k}(m)\right|^{2} \operatorname{trc}\left[\mathscr{K}^{(j)}(m)\right] .
\end{aligned}
$$

If data is not encoded, (42) can be obtained as

$$
b_{k}^{(j+1)}(m)=\frac{\beta_{k}^{(j)}(m)}{\operatorname{trc}\left[\boldsymbol{\kappa}_{n}(m)\left(\boldsymbol{\Sigma}_{\mathbf{h}}^{(j)}+\boldsymbol{\mu}_{\mathbf{h}}^{(j)} \boldsymbol{\mu}_{\mathbf{h}}^{(j)^{H}}\right) \boldsymbol{\kappa}_{k}^{H}(m)\right]} .
$$

It should be noted that (43) is a continuous-valued expression. However, the user data $b_{k}^{(j+1)}(m)$ has a discrete value corresponding to a signal constellation point; hence, at each iteration step, the expression in (43) must be quantized to a signal constellation point closest to itself.

Considering that (32) is a MAP channel estimator, (43) can be interpreted as joint channel estimator and data detector. Also, the quantities $\beta_{k}^{(j)}(m)$ in (40) for each user can be thought as the outputs of successive interference canceller at the $j$-th iteration step. As a result, the proposed iterative algorithm can be called a suboptimal receiver. The flowchart of the algorithm is given as Figure 1.

3.3. Initialization. In order to initialize the proposed SAGE algorithm, the initial values of the channel vector and the data vector must be known. The performance of the algorithm is directly related to the proper selection of these values. The initial value of the channel vector can be obtained by focusing only the training sequence. The resulting signal model, called under-sampled signal model, is

$$
\mathbf{y}_{r}=\Theta_{r} \mathbf{h}_{r}+\mathbf{w}_{r}
$$

$(\cdot)_{r}$ indicates that it is a reduced version of $(\cdot)$. By using wellknown classical channel estimation method, undersampled channel parameters can be obtained as

$$
\widehat{\mathbf{h}}_{r}^{(0)}=\left(\boldsymbol{\Theta}_{r}^{H} \boldsymbol{\Theta}_{r}+\frac{\sigma^{2}}{N} \mathbf{C}_{\mathbf{h}}^{-1}\right)^{-1} \Theta_{r}^{H} \mathbf{y}_{r}
$$

Hence, full channel knowledge can easily be obtained using an interpolation technique.

To detect the initial data symbols, the same classic estimation method MMSE can be exploited. A new signal model is required to reduce the complexity of MMSE based initial data detection method. Once the pilot symbols have been removed, the received signal can be written as

$$
\widetilde{\mathbf{y}}=\mathbf{G}^{(0)} \mathfrak{b}_{k}^{(0)}+\widetilde{\mathbf{n}},
$$

where $\widetilde{\mathbf{y}}$ and $\mathfrak{b}_{k}^{(0)}$ are dimensionally reduced versions of $\mathbf{y}$ and $\mathfrak{b}_{n}$, whereas each entry of noise vector $\widetilde{\mathbf{n}}$ is $\widetilde{\mathbf{n}}(m)=$ $\sum_{n=1, n \neq k} b_{n}(m) \boldsymbol{\rho}_{n k} \mathbf{F}(m) \mathbf{g}(m), \forall m$, and $\mathbf{G}^{(0)} \in \mathbb{C}^{M \times M}$ is a diagonal matrix, each entry of which is $\boldsymbol{\rho}_{k k} \mathbf{F}(m) \mathbf{g}(m)$. From the observation equation (46), initial estimate of data vector for user $k$ can be found as

$$
\widehat{\mathbf{b}}_{k}^{(0)}=\mathbf{G}^{(0)^{H}}\left(\mathbf{G}^{(0)} \mathbf{G}^{\left(0^{H}\right.}+\frac{\sigma^{2}}{N} \mathbf{I}_{M}\right)^{-1} \widetilde{\mathbf{y}}
$$

It should be noted that finding an initial estimate of data vector for user $k$ does not need matrix inversion since $\mathbf{G}^{(0)}$ is a diagonal matrix.

3.4. Complexity Analysis. Computational complexity analysis of the proposed algorithm will not be exhibited in detail, whereas it is presented roughly. When the proposed algorithm is examined carefully, it can be easily seen that the complex multiplication operations are dominant over whole computational complexity. Thus, finding the multiplication operations while computing the expressions in (37) and (38) will lead to a rough result for the computational complexity of the proposed algorithm. The trace operation in (37) requires $4 N D S$ complex multiplications and $3 N D S$ complex additions. In order to calculate (38), 5NDS complex multiplications and 4NDS complex addition operations are required. As a result, a total of $9 N D S$ complex multiplications and $7 N D S$ complex additions must be performed in each iteration step of the proposed algorithm. By defining number of iterations that the algorithm needs to converge as $J$, total number of complex operations is approximately $O(16 J N D S)$.

Based on this result and keeping low computational requirement of DWHT algorithm in mind, it can be concluded that the proposed algorithm has low computational cost if compared to the existing works in the literature such as $[13,25,26]$. The computational load of the proposed SAGE algorithm is given here roughly; it is possible to find a bit more detailed analysis in [13].

3.5. PAPR Analysis. Just as in OFDM, MC-CDMA systems inherently suffer from the PAPR problem. Since PAPR causes 


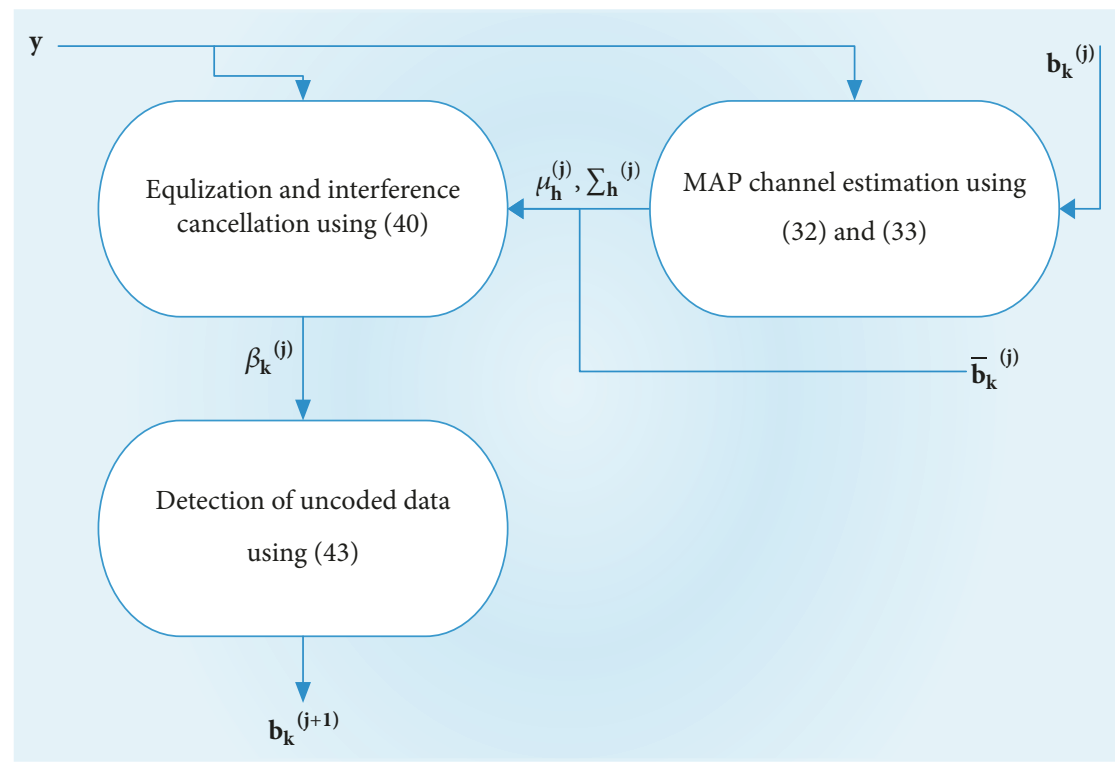

FIGURE 1: The flowchart of the proposed algorithm.

nonlinear distortions at the output of the high power amplifier, the BER or SER performance of the system is reduced. To overcome this problem, linear filters with large dynamic range are required. Such filters increase the system cost considerably and their power efficiency is also very low. The underlying power efficiency problem prevents the use of these filters, since they make the coverage area of a wireless or a mobile communication system inadequate. For all these reasons, many different techniques have been proposed to reduce $\mathrm{PAPR}$, such as clipping and filtering, companding, precoding transform, the partial transmit sequence, coding, and the selective mapping.

In this study, the DWHT method, which is used as a base expansion model to represent time-varying channels with fewer coefficients, is also a transformation with compression capability. The use of DWHT in $[33,34]$ to reduce the PAPR effect encouraged us to examine whether the proposed scheme can reduce PAPR, like a companding method.

The PAPR is defined as the variation of the envelope of a multicarrier signal and is expressed as follows:

$$
\operatorname{PAPR}=\frac{P_{p}}{P_{\mathrm{av}}}=\frac{\max \left|x_{v}\right|^{2}}{(1 / N) \sum_{v=0}^{N-1}\left|x_{v}\right|^{2}},
$$

where $x_{v}$ for $v=0,1, \ldots, N-1$ represents the time samples of an MC-CDMA symbol. $P_{p}$ is peak power of the MC-CDMA signal and $P_{\mathrm{av}}$ is its average power. It is possible to decrease the PAPR value by decreasing the numerator of (48) or by increasing its denominator or both.

After multicarrier modulation, the downlink signal transmitted through the high power amplifier can be written as

$$
s(t)=\mathfrak{R}\left(\sum_{k=1}^{K} \sum_{n=0}^{N-1} b_{k}(t) c_{n}^{(k)} e^{j 2 \pi n\left(t / T_{s}\right)}\right),
$$

where $b_{k}(t)$ is the $k$-th user data and $c_{n}^{(k)}$ is $n$-th chip of $k$ th user. If (48) can be arranged for (49), we can obtain the following upper bound for PAPR using the Cauchy-Schwartz inequality as

$$
\operatorname{PAPR} \leq \frac{2 \max \left\{\sum_{k=1}^{K}\left|\sum_{n=0}^{N-1} c_{n}^{(k)} e^{j 2 \pi n\left(t / T_{s}\right)}\right|^{2}\right\}}{N}
$$

However, the statistical definition of PAPR is more widely used in the literature as the theoretical maximum value of the PAPR in (50) has a low probability of occurrence due to discrepancy between the values of analog and digital PAPRs. The cumulative distribution function (CDF) approach is a common statistical method used for measuring the efficiency of PAPR. However, complementary cumulative distribution function (CCDF) is preferred instead of CDF since CCDF is more suitable for evaluating PAPR. So, we decided to measure the PAPR performance of considered MC-CDMA system using this statistical method. According to [36], power distribution of OFDM signal becomes a central chi-square distribution with 2 degrees of freedom and zero mean. Assuming the variance of one complex sinusoid as $\alpha$, its cumulative distribution can be written as

$$
F(z)=\frac{1}{2 \alpha^{2}} \int_{0}^{z} \exp \left(-\frac{u}{2 \alpha^{2}}\right) d u
$$

Assuming the samples $z$ to be mutually uncorrelated, we can then write the CDF of the PAPR of a data block with Nyquist rate sampling as follows:

$$
\operatorname{Pr}(\operatorname{PAPR} \leq z)=(F(z))^{N}
$$

Since we want to evaluate the PAPR, we need complement of (53) in probabilistic manner. According to [36], it is given 
for QPSK signalling and for large number of subcarriers $(P=$ $N>64)$ as

$$
\begin{aligned}
\operatorname{Pr}(\operatorname{PAPR}>z) & =1-\operatorname{Pr}(\operatorname{PAPR} \leq z)=1-F(z)^{N} \\
& =1-\left(1-e^{-z}\right)^{N} .
\end{aligned}
$$

\section{Computer Simulations}

In this section, Monte-Carlo simulations are carried out to demonstrate the performance of the proposed structure. In the simulations, a downlink MC-CDMA system operating at a frequency of $2.6 \mathrm{GHz}$ is considered. The communication channel is modeled as a channel with an exponentially decreasing power delay profile and a length $S=4$. The power components for each path of the channel are modeled as 0 , $-5,-10$, and $-15 \mathrm{~dB}$ and the path delays as $0, T_{s}, 2 T_{s}$, and $3 T_{s}$, respectively. We consider a resource block size of $M=6$ with two pilot symbols, that is, one as preamble and other one as postamble. It is chosen to be particularly short in order to be able to track the channel in the presence of high mobility. In simulations where symbol error rates are examined, the speed of the mobile unit is set to $295 \mathrm{~km} / \mathrm{h}\left(f_{D} T=0.05\right)$ unless otherwise stated. It is assumed that $K=8$ mobile users are active in the system. For convenience, the number of subcarriers and the length of the Walsh type spreading code are assumed to be equal and each of them is chosen as $P=N=256$.

Figure 2 shows that the variation of the channel's mean squared error for different basis expansion methods when the channel is represented by a different number of coefficients. As can be seen from this figure, the best performance among these curves belongs to discrete Karhunen-Loeve transform (DKLT) BEM that is the optimal one. However, it is well known that this method is sensitive to variations in the channel and therefore needs exact channel statistics. Moreover, the computational cost of this method is high. The performance of the polynomial BEM (Poly) is the next best one, but it is affected from the Doppler spread. Moreover, the order of the polynomials seriously affects the performance of this method. The performances of the rest two methods are almost the same. Although compression capacity of DCT is quite high (DCT is perfectly bandlimited and low-pass), the DCT-BEM has the same structure as the complex exponential (CE) BEM, so it has similar problems. For this reason, it is not suitable for the representation of time-varying channels like CE-BEM. Considering performance close to DCT in Figure 2, the proposed method, DWHT, can be considered as a preferred BEM method in mobile units because of its advantages in hardware implementation due to the small amount of computational burden. In addition, Figure 3 shows that, despite the increasing values of the Doppler spread, the proposed DWHT method does not exhibit any sensitivity to it. With increasing mobility, DWHT exhibits reasonably performance reduction such as the methods DCT and DKLT.

The SER performances of the proposed estimator are shown in Figure 4 when the speed of the mobile unit is $295 \mathrm{~km} / \mathrm{h}$ and $590 \mathrm{~km} / \mathrm{h}$. In this figure, the proposed method SAGE DWHT is compared with methods of MMSE which

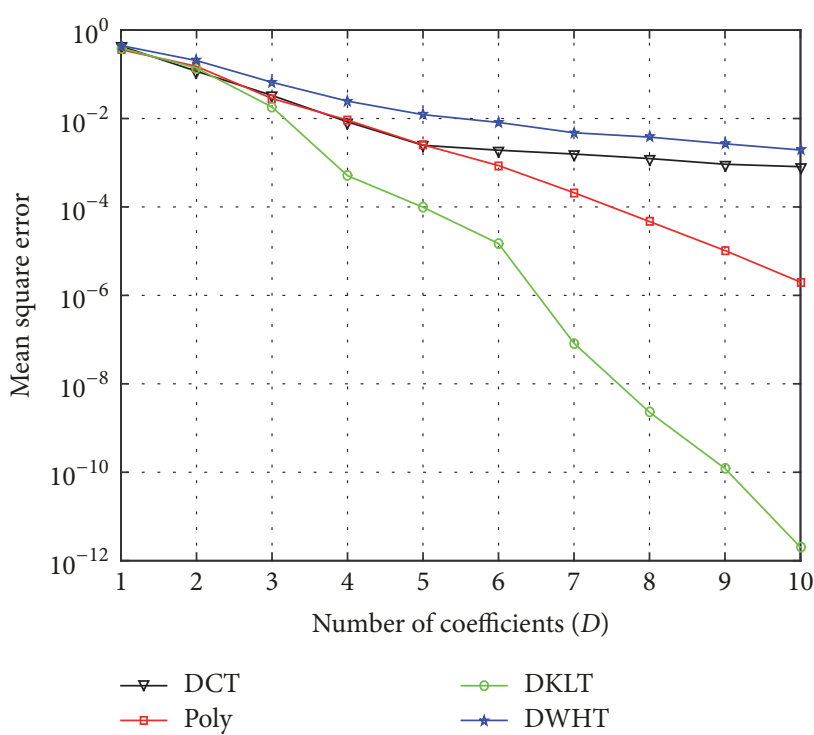

FIgURE 2: Average MSE of channel estimation versus number of coefficients.

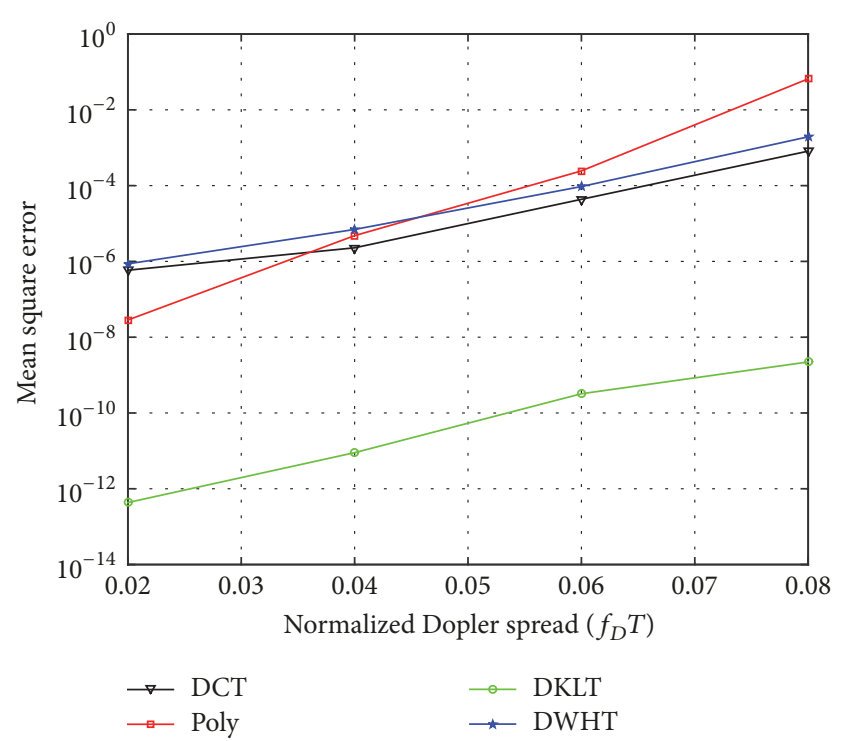

FIGURE 3: Average MSE of channel estimation for several normalized Doppler spread.

estimates data and channel separately, the proposed algorithm using DCT as a BEM (SAGE DCT), and the proposed algorithm having perfect channel knowledge (SAGE for perfect CSI). It is observed that the proposed method provides a better performance than MMSE and is very close to the performance of the SAGE DCT. Given the competitive performance exhibited, it is obvious that the proposed method will be advantageous for mobile units since DWHT and inverse DWHT transformations can only be performed by simply addition and subtraction operations. Also, note that, as the velocity increases, the rapidly varying channel not only destroys the orthogonality but also provides the receiver with time-diversity. In case of perfect CSI (SAGE for perfect 


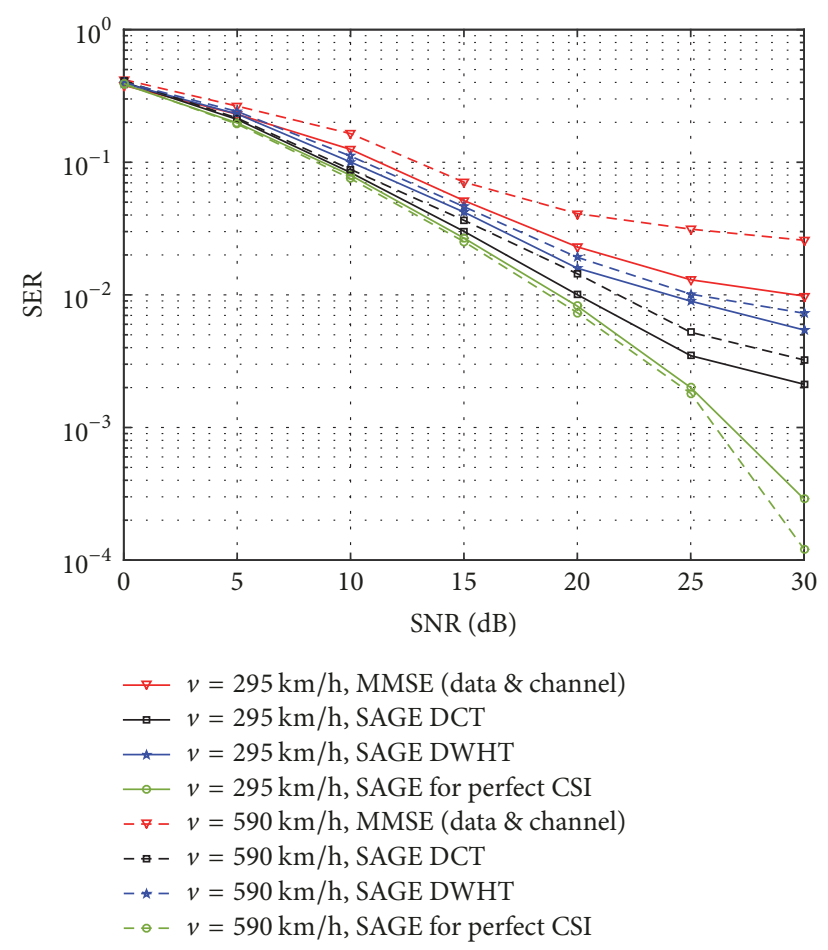

FIGURE 4: Performance comparison of the proposed algorithm with different methods in terms of SER for $P=256, S=4, f_{D} T=$ $0.05(295 \mathrm{~km} / \mathrm{h}), f_{D} T=0.0999(590 \mathrm{~km} / \mathrm{h})$, and QPSK modulation.

CSI), the proposed algorithm performs better although the speed is increased. This can be seen clearly in Figure 4 from SAGE for perfect CSI $(v=295 \mathrm{~km} / \mathrm{h})$ curve and SAGE for perfect CSI $(v=590 \mathrm{~km} / \mathrm{h})$ curve. However, if the channel is not known perfectly, this gain from the diversity disappears due to channel estimation error.

In Figure 5, the SER performance curves of the proposed method are shown for different modulation levels, and each of which is compared to a corresponding lower bound when the receiver has perfect CSI. From the curves, it is concluded that there is no significant loss in SER performance of the proposed method at low and medium values of SNR even if there is no channel information at the receiver side.

Figure 6 shows the convergence rate of our iterative algorithm for QPSK modulation and for several values of SNR. Depending on the initial values and $S N R$, the convergence of our proposed algorithm takes 3 or 4 iterations. The fact that the mobile unit has different speeds does not have a significant effect on the convergence rate of the algorithm. Consequently, a separate figure showing the convergence rate of the algorithm for different speeds is not presented.

Finally, we examined whether the proposed basis expansion method is effective on a PAPR like other companding techniques. Figure 7 depicts the CCDF performances of original MC-CDMA system and MC-CDMA with DWHT. While using QPSK signalling and choosing $P=256$ for the proposed synchronous MC-CDMA system, we have observed that the use of DWHT reduces the PAPR significantly with about $1.5 \mathrm{~dB}$ for $\mathrm{CCDF}(\mathrm{PAPR}) \leq 10^{-3}$.

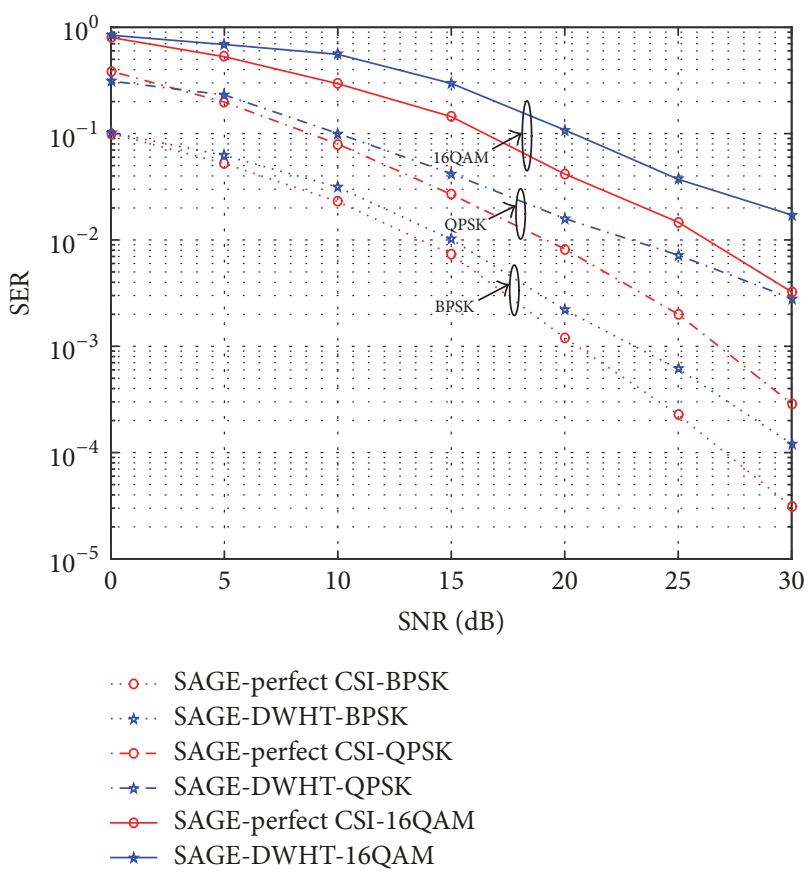

FIGURE 5: SER performance of the proposed algorithm for various modulation schemes.

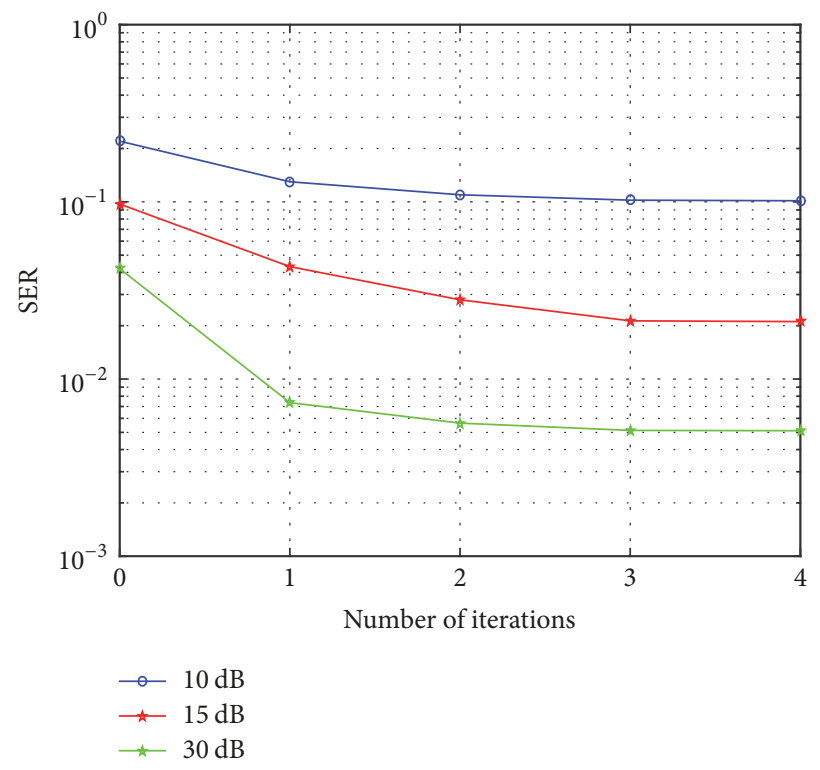

FIGURE 6: Convergence of SER with respect to the number of iterations.

\section{Conclusions}

In this work, we propose an iterative joint channel estimation, equalization, and data detection method for downlink communication of MC-CDMA systems communicating with frequency selective and time-varying channels. Closed form expressions for the data detection of the proposed SAGEbased algorithm have been derived. Since the main goal of the 


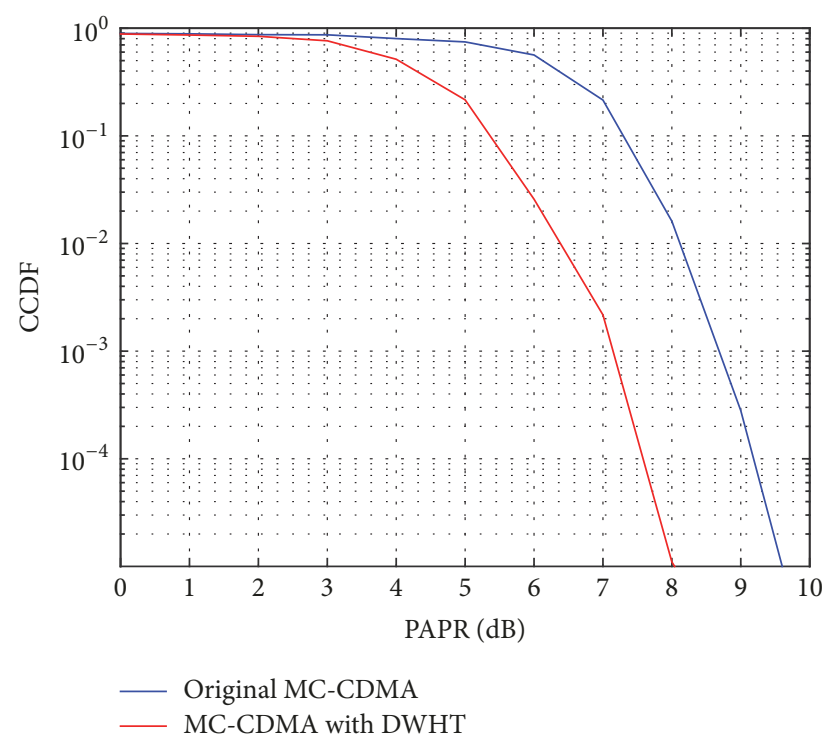

FIGURE 7: CCDFs with/without DWHT versus several $\mathrm{dB}$ values of PAPR for QPSK signalling.

work is to provide a method with less computational complexity, the discrete Walsh-Hadamard base functions have been used to represent rapidly time-varying channels in downlink communications. DWHT has been chosen in particular because it has a low computational cost and it can be easily implemented in hardware with simple addition and subtraction operations. It has been observed that, depending on the normalized Doppler frequency, it is sufficient to represent the channel satisfactorily with a few expansion coefficients. Moreover, no statistical information is needed about the input data. Also, it has a simple implementation, initialization process, and low computational burden if compared to VBLAST, WIMAX, and LTE. This is because VBLAST requires computing inverse of matrices whose dimension equals the number of subcarriers used in OFDM, and the number of subcarriers in the system based on WIMAX and LTE is much greater than one in the normal multicarrier case. Since the DWHT method used in the proposed receiver is a compression capable method, it provides a reduction in PAPR by acting as a companding method.

From the simulation results, it can be seen that both SER and channel estimation performances of the proposed method is not excellent but satisfactory. Thanks to its low computational complexity, it can easily be implemented on a mobile unit with an acceptable performance. Moreover, if a coding scheme is adopted to the proposed method, the performance of it can considerably increase. Finally, from Figure 7, the proposed method achieves the same SER performance with an average PAPR of approximately $1.5 \mathrm{~dB}$ lower.

\section{Conflicts of Interest}

The authors declare that there are no conflicts of interest regarding the publication of this paper.

\section{References}

[1] K. Fazel and S. Kaiser, "Multi-Carrier and Spread Spectrum Systems: From OFDM and MC-CDMA to LTE and WiMAX: Second Edition," John Wiley \& Sons, pp. 1-360, 2008.

[2] K. A. Suharja, R. P. Astuti, L. Meylani, and A. Fahmi, "Enhancement of MC-CDMA performance system using rotated modulation," in Proceedings of the 5th IEEE International Conference on Communication, Network, and Satellite, COMNETSAT 2016, pp. 14-17, Indonesia, December 2016.

[3] J. Wang and Y. Wang, "Analysis performance of MC-CDMA communication system based on improved Chebyshev sequence," in Proceedings of the 2nd IEEE International Conference on Computer and Communications, ICCC 2016, pp. 2277-2280, China, October 2016.

[4] C. Kotchasarn, "Bit error rate expression of MC-CDMA system in $\alpha-\mu$ Fading channel," in Proceedings of the 19th International Conference on Advanced Communications Technology, ICACT 2017, pp. 128-132, Republic of Korea, February 2017.

[5] M. Li, C. Liu, and S. V. Hanly, "Precoding for the sparsely spread MC-CDMA downlink with discrete-alphabet inputs," IEEE Transactions on Vehicular Technology, vol. 66, no. 2, pp. 11161129, 2017.

[6] V. Kuhn, "Combined MMSE-PIC in coded OFDM-CDMA systems," in Proceedings of the GLOBECOM '01. IEEE Global Telecommunications Conference, pp. 231-235, San Antonio, TX, USA, 2001.

[7] H. Doğan, E. Panayirci, and H. A. Çirpan, "EM-based MAP channel estimation and data detection for downlink MCCDMA systems," in Proceedings of the 2007 IEEE Wireless Communications and Networking Conference, WCNC 2007, pp. 227-231, China, March 2007.

[8] H. Doğan, E. Panayirci, H. A. Çirpan, and B. H. Fleury, "MAP channel-estimation-based PIC receiver for downlink MCCDMA systems," EURASIP Journal on Wireless Communications and Networking, vol. 2008, Article ID 570624, 2008.

[9] A. Kocian and B. H. Fleury, "EM-based joint data detection and channel estimation of DS-CDMA signals," IEEE Transactions on Communications, vol. 51, no. 10, pp. 1709-1720, 2003.

[10] E. Panayirci, H. Dogan, H. A. Cirpan, and B. H. Fleury, "Joint data detection and channel estimation for uplink MC-CDMA systems with EM-Based algorithms," in in Proceedings of the International Symposium on Communication Theory and Applications, ISCTA07, Ambleside, Lake District, UK, July 2007.

[11] E. Panayirci, H. Doğan, H. A. Çirpan, A. Kocian, and B. H. Fleury, "Iterative joint data detection and channel estimation for uplink MC-CDMA systems in the presence of frequency selective channels," Physical Communication, vol. 3, no. 2, pp. 87-96, 2010.

[12] A. A. D'Amico, U. Mengali, and M. Morelli, "Channel estimation for the uplink of a DS-CDMA system," IEEE Transactions on Wireless Communications, vol. 2, no. 6, pp. 1132-1137, 2003.

[13] E. Panayirci, H. Senol, and H. V. Poor, "Joint channel estimation, equalization, and data detection for OFDM systems in the presence of very high mobility," IEEE Transactions on Signal Processing, vol. 58, no. 8, pp. 4225-4238, 2010.

[14] N. Kabaoğlu, "SAGE based suboptimal receiver for downlink MC-CDMA systems," IEEE Communications Letters, vol. 15, no. 12, pp. 1381-1383, 2011.

[15] O. H. Salim, A. A. Nasir, H. Mehrpouyan, W. Xiang, S. Durrani, and R. A. Kennedy, "Channel, phase noise, and frequency offset in OFDM systems: joint estimation, data detection, 
and hybrid Cramér-Rao lower bound," IEEE Transactions on Communications, vol. 62, no. 9, pp. 3311-3325, 2014.

[16] A. Assra, W. Hamouda, and A. Youssef, "EM-based joint channel estimation and data detection for MIMO-CDMA systems," IEEE Transactions on Vehicular Technology, vol. 59, no. 3, pp. 1205-1216, 2010.

[17] R. Prasad, C. R. Murthy, and B. D. Rao, "Joint channel estimation and data detection in MIMO-OFDM systems: a sparse Bayesian learning approach," IEEE Transactions on Signal Processing, vol. 63, no. 20, pp. 5369-5382, 2015.

[18] A. Kalakech, L. Brunel, M. Berbineau, and D. Mottier, "A novel OFDM power based estimation for dynamic channel tracking in downlink lte," in Proceedings of the 76th IEEE Vehicular Technology Conference, VTC Fall 2012, Canada, September 2012.

[19] X. Cai, X. Yin, and A. P. Yuste, "Direction-of-arrival estimation using single antenna in high-speed-train environments," in Proceedings of the 10th European Conference on Antennas and Propagation, EuCAP 2016, Switzerland, April 2016.

[20] T. Zemen, H. Hofstetter, and G. Steinbock, "Successive slepian subspace projection in time and frequency for time-variant channel estimation," in Proceedings of the 14th IST Mobile and Wireless Communication Summit, Germany, 2005.

[21] H. Wang and T. Li, "Channel tracking and signal detection for MC-CDMA in a time-varying environment," IEEE Transactions on Vehicular Technology, vol. 56, no. 6 I, pp. 3613-3620, 2007.

[22] J. Wehinger, T. Zemen, A. Kocian, and B. H. Fleury, "Low-complexity joint data detection and channel estimation in timevarying flat-fading channels within the SAGE framework," in Proceedings of the Newcom-Acorn Joint Workshop, pp. 20-22, Vienna, Austria, 2006.

[23] M. Ahmed and N. M. Khan, "Model based approach for timevarying channel estimation in DS-CDMA systems using nonisotropic scattering environment," in Proceedings of the 2009 International Conference on Emerging Technologies, ICET 2009, pp. 62-65, Pakistan, October 2009.

[24] A. Akan, E. Önen, and L. F. Chaparro, "Time-frequency based channel estimation for high-mobility OFDM systems-part I: MIMO case," EURASIP Journal on Advances in Signal Processing, vol. 2010, Article ID 549197, 8 pages, 2010.

[25] O.-E. Barbu, C. N. Manchon, C. Rom, T. Balercia, and B. H. Fleury, "OFDM receiver for fast time-varying channels using block-sparse bayesian learning," IEEE Transactions on Vehicular Technology, vol. 65, no. 12, pp. 10053-10057, 2016.

[26] D. Hu, X. Wang, and L. He, "A new sparse channel estimation and tracking method for time-varying OFDM systems," IEEE Transactions on Vehicular Technology, vol. 62, no. 9, pp. 46484653, 2013.

[27] H. Xie, F. Gao, S. Zhang, and S. Jin, "Spatial-temporal BEM and channel estimation strategy for massive MIMO time-varying systems," in Proceedings of the 59th IEEE Global Communications Conference, GLOBECOM 2016, USA, December 2016.

[28] L. Deng, Z. Chen, and Y. Zhao, "Basis expansion model for channel estimation in LTE-R communication system," Digital Communications and Networks, vol. 2, no. 2, pp. 92-96, 2016.

[29] A. Chacko and J. Jeevitha, "PAPR reduction techniques for performance improvement in OFDM systems," in Proceedings of the 2014 International Conference on Electronics and Communication Systems, ICECS 2014, India, February 2014.

[30] S. S. Prabhu and P. Premalatha, "A PAPR reduction technique in OFDM transmissions using hierarchical QAM," in Proceedings of the 2016 International Conference on Communication and Signal Processing, ICCSP 2016, pp. 1650-1653, India, April 2016.
[31] G. Kaur, N. Kumar, and B. S. Sohi, "PAPR reduction technique on wavelet based OFDM system by employing multi-level wavelet transform," in Proceedings of the International Conference on Signal Processing, Computing and Control, ISPCC 2015, pp. 215-219, India, September 2015.

[32] A.-K. Ajami and H. A. Artail, "KLT-based PAPR reduction technique for LTE-Advanced uplink with carrier aggregation," in Proceedings of the 11th IEEE International Conference on Wireless and Mobile Computing, Networking and Communications, WiMob 2015, pp. 514-519, UAE, October 2015.

[33] S. Shete, G. Bhide, and M. Jadhav, "WHT and Double WHT: An effective PAPR reduction approach in OFDM," in Proceedings of the 2016 IEEE International Conference on Advances in Electronics, Communication and Computer Technology, ICAECCT 2016, pp. 172-175, India, December 2016.

[34] C. Kang, Y. Liu, M. Hu, and H. Zhang, "A low complexity PAPR reduction method based on FWFT and PEC for OFDM systems," IEEE Transactions on Broadcasting, vol. 63, no. 2, pp. 416425, 2017.

[35] W. C. Jakes, Microwave Mobile Communications, Wiley-IEEE Press, Piscataway, NJ, USA, 1994.

[36] R. van Nee and A. de Wild, "Reducing the peak-to-average power ratio of OFDM,' in Proceedings of the VTC '98. 48th IEEE Vehicular Technology Conference. Pathway to a Global Wireless Revolution, pp. 2072-2076, Ottawa, Ont., Canada, 1998. 


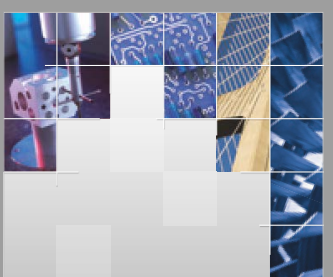

\section{Enfincering}
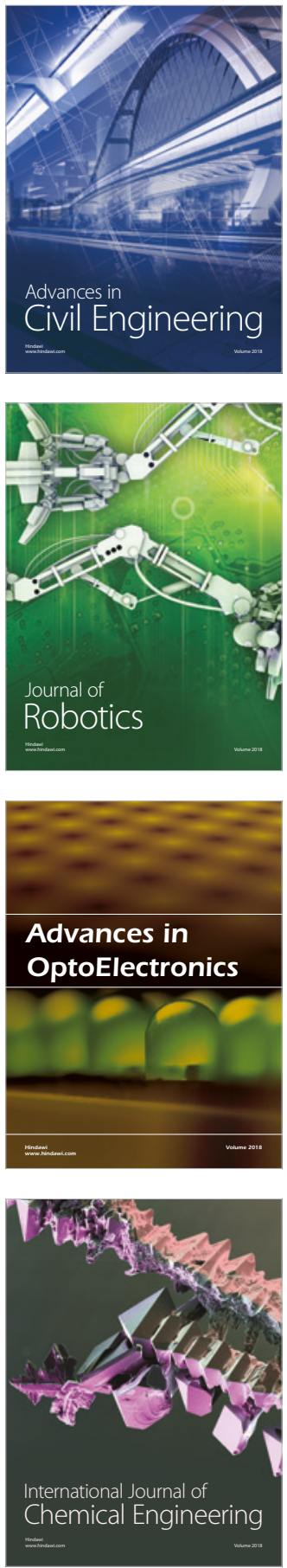

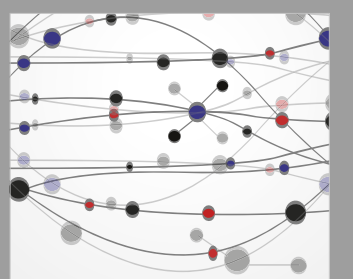

\section{Rotating \\ Machinery}

The Scientific World Journal

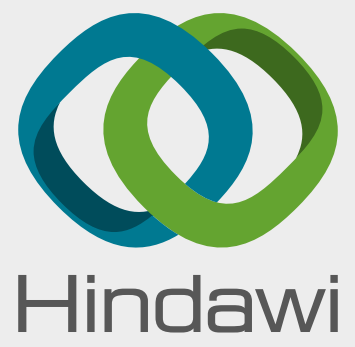

Submit your manuscripts at

www.hindawi.com
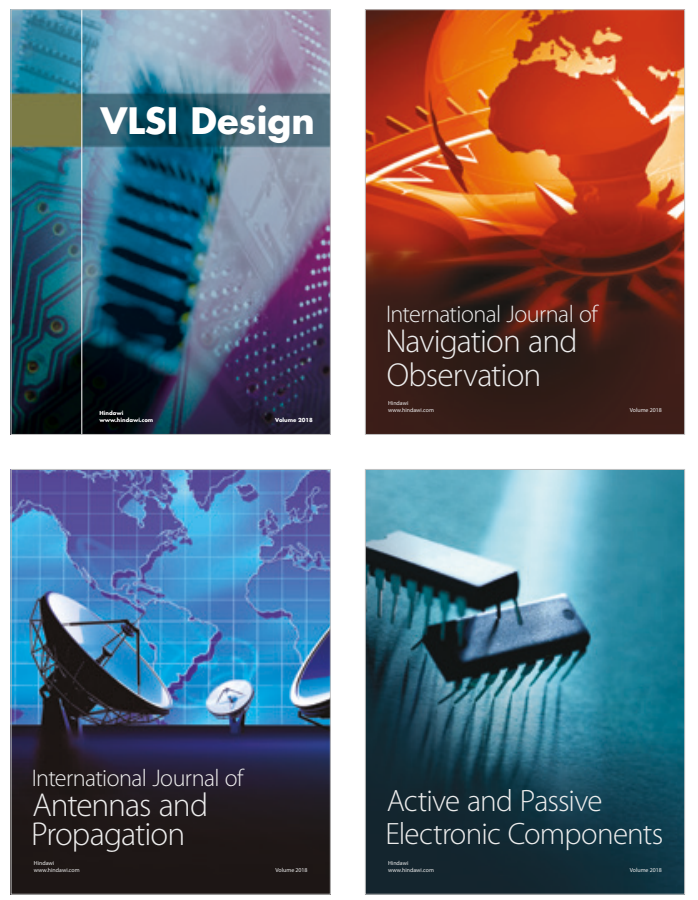
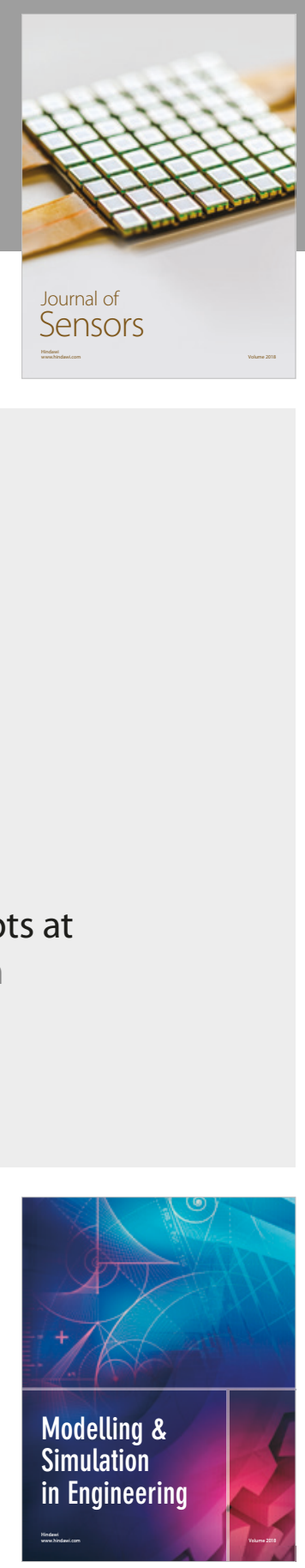

\section{Advances \\ Multimedia}
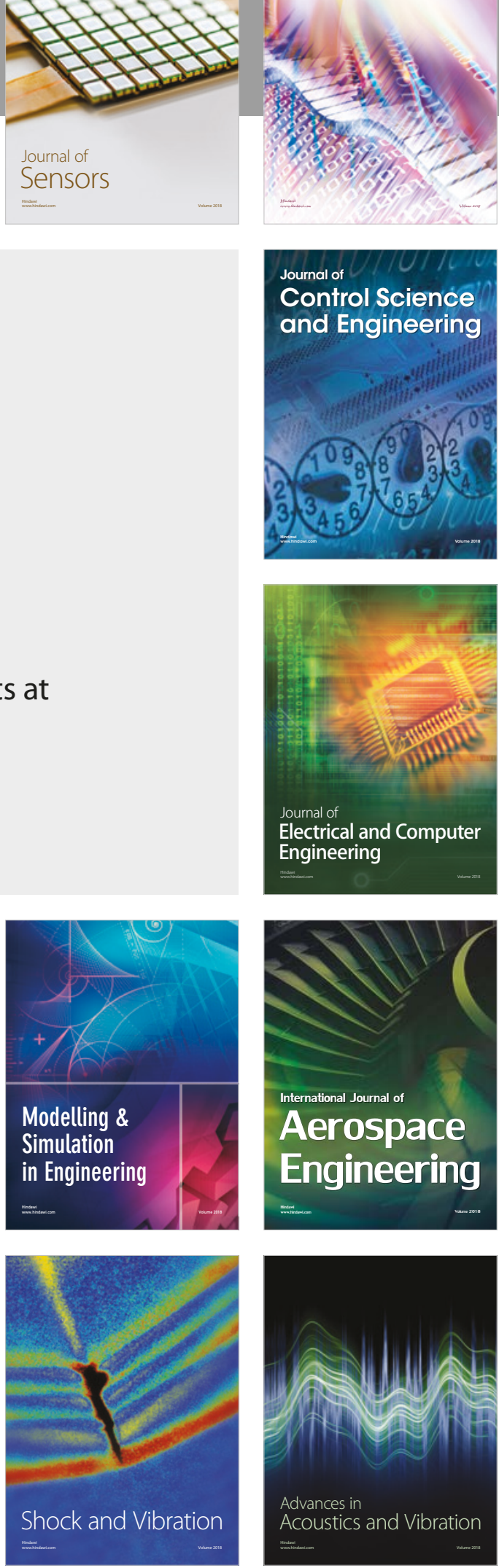